\title{
AUTOMORPHISMS AND DERIVATIONS OF INCIDENCE ALGEBRAS
}

\author{
KENNETH BACLAWSKI ${ }^{1}$
}

\begin{abstract}
This paper studies the derivations and automorphisms of the incidence algebra of a locally finite partially ordered set. Two subspaces are shown to span the space of derivations: the space of inner derivations and the space of derivations associated with the additive functions. An analogous result is shown for the group of automorphisms. A number of dimension calculations are also made.
\end{abstract}

1. Preliminaries. In the study of additive and multiplicative functions on the segments of a (partially) ordered set, it is natural to consider derivations and automorphisms, respectively, of the incidence algebra. In $\S 2$, we study the derivations. In $\S 3$, we give the analogous results for automorphisms. For a more complete description of the notation used see [1].

Let $P$ be a locally finite (partially) ordered set, i.e. for which every segment $[x, y]=\{z \mid x \leqq z \leqq y\}$ is finite. The incidence algebra $I(P)$ of $P$ over a field $K$ is defined as the $K$-algebra of all functions from segments of $P$ into $K$ under the product

$$
f * g(x, y)=\sum_{x \leqslant z \leqslant y} f(x, z) g(z, y),
$$

where we write $f(x, y)$ for $f([x, y])$. The Schur (pointwise) product, $f g(x, y)=f(x, y) g(x, y)$, will also be used. The elements $\delta, e_{x}$ and $\delta_{x y}$ of $I(P)$ are defined in [1]. We also assume that $I(P)$ is given the standard topology induced by the discrete topology on $K$ as defined in [1]. With this topology, $I(P)$ is a topological algebra with respect to either product.

Received by the editors December 15, 1971 and, in revised form, April 12, 1972.

AMS 1970 subject classifications. Primary 06A10, 16A72; Secondary 05A15.

Key words and phrases. Ordered set, partially ordered set, incidence algebra, automorphism, derivation, additive function, multiplicative function.

${ }^{1}$ This work was done while the author was supported by an NSF Graduate Fellowship.

c) American Mathematical Society 1973 
2. Derivations. Let $\mathscr{J}(I(P))$ be the space of $K$-derivations of $I(P)$, i.e. the space of $K$-homomorphisms $D: I(P) \rightarrow I(P)$ satisfying

$$
D(f * g)=f * D(g)+D(f) * g,
$$

for all $f, g \in I(P)$. For any $f \in I(P)$, the inner derivation of $I(P)$ with respect to $f$ is the derivation $d_{f}$ given by $d_{f}(g)=f * g-g * f$. Let $\mathscr{I} \mathscr{D}(I(P))$ denote the space of all inner derivations of $I(P)$.

THEOREM 1. Let $P$ be a locally finite ordered set having $c$ connected components: $P_{1}, \cdots, P_{c}$. Then there is a short exact sequence of $K$-homomorphisms

$$
0 \longrightarrow K^{c} \stackrel{j}{\longrightarrow} I(P) \stackrel{d}{\longrightarrow} \mathscr{I} \mathscr{L}(I(P)) \longrightarrow 0 .
$$

We define $j: K^{c} \rightarrow I(P)$ by $j\left(a_{1}, \cdots, a_{c}\right)=g$, where

$$
\begin{aligned}
g(x, y) & =a_{i}, \quad \text { if } x=y \in P_{i}, \\
& =0, \quad \text { if } x \neq y .
\end{aligned}
$$

The $K$-homomorphism $d: I(P) \rightarrow \mathscr{I} \mathscr{D}(I(P))$ is, of course, given by $d(f)=d_{f}$.

Proof. We clearly need only check the result for $c=1$, i.e. for $P$ connected. In this case, $d$ is obviously a $K$-epimorphism of $I(P)$ onto $\mathscr{I} \mathscr{D}(I(P))$ whose kernel is the center of $I(P)$. Thus $\operatorname{Im}(j)=K \delta \subseteq \operatorname{Ker}(d)$.

Let $f \in \operatorname{Ker}(d)$. Then for $x<y, f(x, y) \delta_{x y}=e_{x} * f * e_{y}=e_{x} * e_{y} * f=0$. Hence $f(x, y)=0$ when $x<y$. Now let $x \leqq y$. Then $f(x, x) \delta_{x y}=\delta_{x x} * f * \delta_{x y}=$ $\delta_{x x} * \delta_{x y} * f=\delta_{x y} * f=\delta_{x y} * \delta_{y y} * f=\delta_{x y} * f * \delta_{y y}=f(y, y) \delta_{x y}$. Therefore, if $x \leqq y, f(x, x)=f(y, y)$. Since $P$ was assumed to be connected, we have $f(x, x)=f(y, y)$ for all $x, y \in P$. Thus $f \in K \delta$. The result now follows.

We call an element $\sigma$ of $I(P)$ additive if, for $x \leqq y \leqq z$,

$$
\sigma(x, z)=\sigma(x, y)+\sigma(y, z) .
$$

For any additive $\sigma \in I(P)$, there is a naturally associated derivation $L_{\sigma}$ defined by $L_{\sigma}(f)=\sigma f$. Let $\mathscr{L} \mathscr{D}(I(P))$ denote the space of all such derivations.

THEOREM 2. If $P$ is a locally finite ordered set, then

$$
\mathscr{D}(I(P))=\mathscr{I} \mathscr{R}(I(P))+\mathscr{L} \mathscr{\mathscr { H }}(I(P)) .
$$

Proof. Let $D \in \mathscr{D}(I(P))$. Set $g=\sum_{p \in P^{\prime}} D\left(e_{p}\right) * e_{p}$. Equivalently, $g(x, y)=D\left(e_{y}\right)(x, y)$ for $x \leqq y$ in $P$; hence $g$ is a well-defined element of $I(P)$.

Let $r, s \in P$. Then $D\left(e_{r} * e_{s}\right) * e_{s}=e_{r} * D\left(e_{s}\right) * e_{s}+D\left(e_{r}\right) * e_{s}$. Therefore

$$
\begin{aligned}
e_{r} * D\left(e_{s}\right) * e_{s} & =-D\left(e_{r}\right) * e_{s}, & & \text { if } r \neq s, \\
& =0, & & \text { if } r=s .
\end{aligned}
$$


Thus for $r \in P$ we have

$$
\begin{aligned}
\left(D-d_{g}\right)\left(e_{r}\right) & =D\left(e_{r}\right)-g * e_{r}+e_{r} * g \\
& =D\left(e_{r}\right)-\sum_{s \in P^{\prime}} D\left(e_{s}\right) * e_{s} * e_{r}+\sum_{s \in I^{\prime}} e_{r} * D\left(e_{s}\right) * e_{s} \\
& =D\left(e_{r}\right)-D\left(e_{r}\right) * e_{r}-\sum_{s \neq r} D\left(e_{r}\right) * e_{s} \\
& =D\left(e_{r}\right)-D\left(e_{r}\right) * e_{r}-D\left(e_{r}\right) *\left(\sum_{s \neq r} e_{s}\right) \\
& =D\left(e_{r}\right)-D\left(e_{r}\right) * e_{r}-D\left(e_{r}\right) *\left(\delta-e_{r}\right)=0 .
\end{aligned}
$$

Since $I(P)$ is a topological algebra and $g$ is well-defined, all the above sums converge.

Now if $x \leqq y$ in $P$, then $e_{x} * I(P) * e_{y}=K \delta_{x y}$. Applying the derivation $D_{0}=D-d_{g}$ to this equation yields $D_{0}\left(\delta_{x y}\right) \in K \delta_{x y}$. Thus there exists some $\sigma \in I(P)$ for which $D_{0}\left(\delta_{x y}\right)=\sigma \delta_{x y}$, for all $x \leqq y$ in $P$. It is easy to verify that $\sigma$ is additive.

To complete the proof, we must show that $D_{0}=L_{\sigma}$. This is not trivial when $P$ is infinite, since there is no a priori reason to expect $D_{0}$ to commute with infinite sums. Let $f \in I(P)$. Then, for $x \leqq y$ in $P$,

$$
D_{0}\left(e_{x} * f * e_{y}\right)=D_{0}\left(f(x, y) \delta_{x y}\right)=f(x, y) \sigma(x, y) \delta_{x y},
$$

and

$$
D_{0}\left(e_{x} * f * e_{y}\right)=e_{x} * D_{0}(f) * e_{y}=D_{0}(f)(x, y) \delta_{x y} .
$$

Thus $D_{0}(f)=\sigma f$. The theorem now follows.

COROllaRY. Every derivation of an incidence algebra with the standard topology is continuous.

Indeed, by Theorem 2 , if $I(P)$ is given any topology such that the three operations $*$, + and - (Schur product) are continuous, then every derivation on $I(P)$ is continuous.

In general, the decomposition $D=d_{g}+L_{\sigma}$ of Theorem 2 is not unique. However, the elements of $\mathscr{I} \mathscr{D}(I(P)) \cap \mathscr{L} \mathscr{L}(I(P))$ have an especially simple form. Let $d_{g}=L_{\sigma}$. Now in the standard topology of $I(P)$, the sum $\sum_{r: s} g(r, s) \delta_{r s}$ converges to $g$. Thus, for $p \leqq q$ in $P$,

$$
\begin{aligned}
\sigma(p, q) \delta_{p q} & =L_{\sigma}\left(\delta_{p q}\right)=d_{g}\left(\delta_{p q}\right)=g * \delta_{p q}-\delta_{p q} * g \\
& =\sum_{r \leqq s} g(r, s)\left(\delta_{r s} * \delta_{p q}-\delta_{p q} * \delta_{r s}\right) \\
& =\sum_{r<p} g(r, p) \delta_{r q}-\sum_{q<s} g(q, s) \delta_{p s}+(g(p, p)-g(q, q)) \delta_{p q} .
\end{aligned}
$$


Thus $g(p, q)=0$ for $p<q$, and $\sigma(p, q)=g(p, p)-g(q, q)$ for $p \leqq q$. Conversely, given any $g \in I(P)$ such that $g(p, q)=0$ for $p<q$, then $\sigma(p, q)=$ $g(p, p)-g(q, q)$ defines an additive element of $I(P)$ for which $L_{\sigma}=d_{g}$. If $P$ has a 0 or 1 , it is easy to verify that every additive element $\sigma$ of $I(P)$ has this form. Thus, in this case, every derivation of $I(P)$ is an inner derivation.

If $P$ is finite, it is clear that the dimension of $\mathscr{I} \mathscr{Z}(I(P)) \cap \mathscr{L} \mathscr{L}(I(P))$ is $q-c$, where $q$ is the cardinality of $P$ and $c$ is the number of connected components of $P$. We have therefore shown one part of the following theorem. The remaining parts use the notation employed in Stanley [4] and are proved in a manner similar to that of the results there $[\mathbf{4}$, Theorem 2].

THEOREM 3. If $P$ is a finite ordered set, then

$$
\begin{aligned}
& \operatorname{dim}_{K} \mathscr{I} \mathscr{T}(I(P)) \cap \mathscr{L} \mathscr{Z}(I(P))=q-c, \\
& \operatorname{dim}_{K} \mathscr{L} \mathscr{P}(I(P))=p-t, \\
& \operatorname{dim}_{\kappa} \mathscr{L} \mathscr{L}(I(P)) \mid \mathscr{I} \mathscr{\mathscr { Z }}(I(P)) \cap \mathscr{L} \mathscr{Y}(I(P))=r-t, \\
& \operatorname{dim}_{K} \mathscr{P}(I(P))=\operatorname{dim}_{K} \mathscr{I} \mathscr{\mathscr { I }}(I(P))+r-t=\operatorname{dim}_{K} I(P)-c+r-t .
\end{aligned}
$$

The last equality is a consequence of Theorem 1.

3. Automo:phisms. Let $K^{*}$ and $I(P)^{*}$ denote the multiplicative subgroups of $K$ and $I(P)$ respectively. The group of all $K$-automorphisms of $I(P)$ is denoted $\mathscr{A}(I(P))$. For $f \in I(P)^{*}$, the inner automorphism of $I(P)$ with respect to $f$ is given by $i_{f}(g)=f * g * f^{-1}$. The group of all inner automorphisms of $I(P)$ is a normal subgroup of $\mathscr{S}(I(P))$ which we denote by $\mathscr{I}(I(P))$. The following theorem is proved in precisely the same manner as Theorem 1.

THEOREM 4. Let $P$ be a locally finite ordered set having c connected components: $P_{1}, \cdots, P_{c}$. Then there is a short exact sequence of homomorphisms

$$
1 \longrightarrow\left(K^{*}\right)^{*} \stackrel{j}{\longrightarrow} I(P)^{*} \stackrel{i}{\longrightarrow} \mathscr{I}(I(P)) \longrightarrow 1
$$

The homomorphism $j:\left(K^{*}\right)^{c} \rightarrow I(P)^{*}$ is given by the restriction of the map $j: K^{c} \rightarrow I(P)$ in Theorem 1 . The homomorphism $i: I(P)^{*} \rightarrow I(I(P))$ is given by $i(f)=i_{f}$.

An element $v$ of $I(P)$ is multiplicatice if, for $x \leqq y \leqq z$,

$$
v(x, z)=v(x, y) v(y, z) \in K^{*} .
$$

In particular, $v \in I(P)^{*}$. For any multiplicative element $v$, there is an automorphism $L_{v}$ of $I(P)$ defined by $L_{v}(f)=v f$. Let $\mathscr{L}(I(P))$ denote the group of all such automorphisms. 
Let $\mathscr{A}(P)$ denote the automorphism group of $P$. Given $\alpha \in \mathscr{A}(P)$, we can associate an automorphism (also denoted $\alpha$ ) of $I(P)$ defined by $\alpha(f)(x, y)=f(\alpha x, \alpha y)$, for all $f \in I(P)$ and $x \leqq y$ in $P$.

THEOREM 5. If $P$ is a locally finite ordered set, then $\mathscr{A}(I(P))$ is generated by. $\mathscr{I}(I(P)), \mathscr{L}(I(P))$ and $\mathscr{A}(P)$ in the sense that if $A \in \mathscr{A}(I(P))$, then there are $i_{f} \in \mathscr{I}(I(P)), L_{v} \in \mathscr{L}(I(P))$ and $\alpha \in \mathscr{A}(P)$ such that $A=i_{f} \circ L_{v} \circ \alpha$. In general, $\alpha$ is unique, but $i_{f}$ and $L_{v}$ need not be.

Proof. The proof follows Stanley's Theorem 2 [4], where the result is proved in the finite case.

Let $A \in \mathscr{A}(I(P))$. Since $\left\{e_{x} \mid x \in P\right\}$ is a maximal set of primitive orthogonal idempotents of $I(P)$, the same is true of $\left\{A\left(e_{x}\right) \mid x \in P\right\}$. By Theorem 1 of [4], there is a unique $\alpha \in \mathscr{A}(P)$ such that for all $x \in P,\left(A \circ \alpha^{-1}\right)\left(e_{x}\right)-$ $e_{x} \in J=\{g \in I(P) \mid g(y, y)=0$ for all $y \in P\}$. If $i_{f} \in \mathscr{I}(I(P))$ and $L_{v} \in \mathscr{L}(I(P))$, then

$$
\left(i_{f} \circ L_{v}\right)(g)(x, x)=g(x, x),
$$

for all $g \in I(P)$ and $x \in P$. It therefore follows that in any decomposition $A=i_{f} \circ L_{v} \circ \alpha, \alpha$ is unique.

Set $f=\sum_{x \in I^{\prime}}\left(A \circ \alpha^{-1}\right)\left(e_{x}\right) * e_{x}$. Then $f$ is an element of $I(P)^{*}$ for which $f * e_{x} * f^{-1}=\left(A \circ \alpha^{-1}\right)\left(e_{x}\right)$, for all $x \in P$. Therefore, $A_{0}=i_{f}^{-1} \circ A \circ \alpha^{-1}$ fixes all the $e_{x}$. Since $e_{x} * I(P) * e_{y}=K \delta_{x y}, A_{0}\left(\delta_{x y}\right) \in K \delta_{x y}$. Let $v \in I(P)$ be defined by $v(x, y)=A_{0}\left(\delta_{x y}\right)(x, y)$. It is easy to check that $v$ is multiplicative.

It remains to check that $A_{0}=L_{v}$. Let $h \in I(P)$ and $x \leqq y$. Then, as in Theorem 2,

$$
A_{0}\left(e_{x} * h * e_{y}\right)=A_{0}\left(h(x, y) \delta_{x y}\right)=h(x, y) v(x, y) \delta_{x y},
$$

and

$$
A_{0}\left(e_{x} * h * e_{y}\right)=e_{x} * A_{0}(h) * e_{y}=A_{0}(h)(x, y) \delta_{x y} .
$$

Hence $A_{0}(h)=v h$, and so $A_{0}=L_{v}$. The theorem now follows.

It is trivial to check that the order of the factors in the decomposition given by Theorem 5 is not the only possible one. For example, if $L_{v} \in$ $\mathscr{L}(I(P))$ and $\alpha \in \mathscr{A}(P)$, then $\tau=\alpha^{-1}(\nu)$ is multiplicative and $L_{v} \circ \alpha=\alpha \circ L_{r}$.

COROllaRY. Every automorphism of an incidence algebra with the standard topology is. continuous.

Indeed, if $I(P)$ is given any topology such that $*,+, \cdot$ and the elements of $\mathscr{A}(P)$ are continuous, then every automorphism (as well as every derivation) of $I(P)$ is continuous. In general, even if $P$ is finite and *, + , and $\cdot$ are continuous, it is possible for an element of $\mathscr{A}(P)$ not to be continuous. 
As in the case of derivations, the elements of $\mathscr{I}(I(P)) \cap \mathscr{L}(I(P))$ have a particularly simple form. Namely, $L_{v}=i_{f}$ if and only if $f(x, y)=0$ for $x<y$ and $v(x, y)=f(x, x) f(y, y)^{-1}$ for $x \leqq y$. If $P$ has a 0 or 1 , every multiplicative element has this form. In this case, $\mathscr{A}(I(P)) / \mathscr{I}(I(P)) \cong \mathscr{A}(P)$.

The author is indebted to Professor Gian-Carlo Rota for several conversations on this subject.

\section{REFERENCES}

1. P. Doubilet, G.-C. Rota and R. Stanley, The idea of generating function, Proc. Sixth Berkeley Sympos. on Math. Statist. and Probability, vol. II, Univ. of California Press, Berkeley, Calif. (to appear).

2. C. L. Liu, Introduction to combinatorial mathematics, McGraw-Hill, New York, 1968. MR 38 \#3154.

3. G.-C. Rota, On the foundations of combinatorial theory. I. Theory of Möbius functions, Z. Wahrscheinlichkeitstheorie und Verw. Gebiete 2 (1964), 340-368. MR 30 \#4688.

4. R. P. Stanley, Structure of incidence algebras and their automorphism groups, Bull. Amer. Math. Soc. 76 (1970), 1236-1239. MR 41 \#8319.

Department of Mathematics, Harvard University, Cambridge, Massachusetts 02138 